\title{
Studies on the Axial Position of the Decelerator in Traveling Wave Direct Energy Converter ${ }^{*)}$
}

\author{
Tomohiro KATSURA, Takahiro WAKAIZUMI, Hiromasa TAKENO, \\ Kazuya ICHIMURA $^{1)}$ and Yousuke NAKASHIMA ${ }^{1)}$ \\ Department of Electrical and Electronic Engineering, Kobe University, \\ 1-1 Rokkodai-cho, Nada-ku, Kobe 657-8501, Japan \\ 1) Plasma Research Center, University of Tsukuba, 1-1-1 Tennodai-cho, Tsukuba 305-8572, Japan
}

(Received 30 November 2015 / Accepted 19 January 2016)

\begin{abstract}
In $\mathrm{D}^{3}{ }^{3} \mathrm{He}$ fusion, most of fusion energy is carried by created protons as kinetic energy, so direct energy conversion can be applied. A traveling wave direct energy converter was proposed as an energy recovering system for these protons, which was composed of a modulator and a decelerator. The axial position of the decelerator is one of the important device parameters for both energy conversion efficiency and device size. The best position for conversion efficiency was considered to be the bunching position at which proton density is the highest, but it was not examined well. In this study, we investigated the dependence of deceleration efficiency on the axial position of the decelerator by using simulation experiments and numerical calculations. The results show that the bunching position is not necessarily the optimum one for conversion efficiency.
\end{abstract}

(c) 2016 The Japan Society of Plasma Science and Nuclear Fusion Research

Keywords: advanced fusion, direct energy conversion, TWDEC

DOI: $10.1585 / \mathrm{pfr} .11 .2405013$

\section{Introduction}

In $\mathrm{D}^{3}{ }^{3} \mathrm{He}$ fusion, neutrons are hardly created, and most of fusion energy is carried by created protons as kinetic energy, so direct energy conversion can be applied. However, energy of these protons is $14.7 \mathrm{MeV}$ and corresponding voltage necessary for a conventional electrostatic converter exceeds an engineering limit. For this issue, a traveling wave direct energy converter (TWDEC) was proposed as an energy recovering system for these protons [1]. A TWDEC is composed of a modulator and a decelerator. Incident proton beam is velocity-modulated by radio frequency (RF) field in the modulator and bunched in the downstream. The beam is guided to the decelerator which consists of a lot of electrodes aligned in the direction of the beam and an external circuit to which the electrodes are connected. When the bunched beam passes through these electrodes, the induced current in the circuit excites a traveling wave, the field of which decelerates the protons. The decreased kinetic energy of protons is converted into electricity in the external circuit.

The axial position of the decelerator is one of the important device parameters for both energy conversion efficiency and device size. When the decelerator is settled near the modulator, the device can be compact, but the efficiency cannot be high. The best position for conversion efficiency is considered to be the bunching position at

author'se-mail: takeno@eedept.kobe-u.ac.jp

*) This article is based on the presentation at the 25 th International Toki Conference (ITC25). which proton density is the highest. In the previous study, the authors conducted simulation experiments and numerical calculations by setting a decelerator at the bunching position [2]. The highest experimental record of the efficiency was obtained, however, the optimum axial position was not examined.

In this study, we investigate the dependence of deceleration efficiency on the axial position of the decelerator. By using simulation experiments and numerical calculations, we examine the efficiency by changing the distance between the modulator and the decelerator, and discuss the optimum position of the decelerator based on the results.

In Sec. 2, the experimental device is explained. In Sec. 3, not only experimental and numerical results, but also discussion on the axial position of the decelerator is shown. In Sec. 4, conclusion of the paper is given.

\section{Experimental Device}

In Fig. 1, a schematic view of the device used in the experiment is given. It consists of an ion source, a modulator, a decelerator, and a measurement section.

To use helium ions instead of protons, helium plasma is produced by an application of a static magnetic field and an RF power of $15 \mathrm{MHz}$ in the ion source of a glass tube. The RF power is supplied with amplitude modulation by continuous rectangular pulse of $1 \mathrm{kHz}$ and a duty of $50 \%$. By applying high voltage $\left(V_{\text {ex }}\right)$ to an extraction electrode, helium ions are extracted to the metal chamber. In this study, $V_{\mathrm{ex}}$ is usually set to be $3.2 \mathrm{kV}$. A convergence elec- 


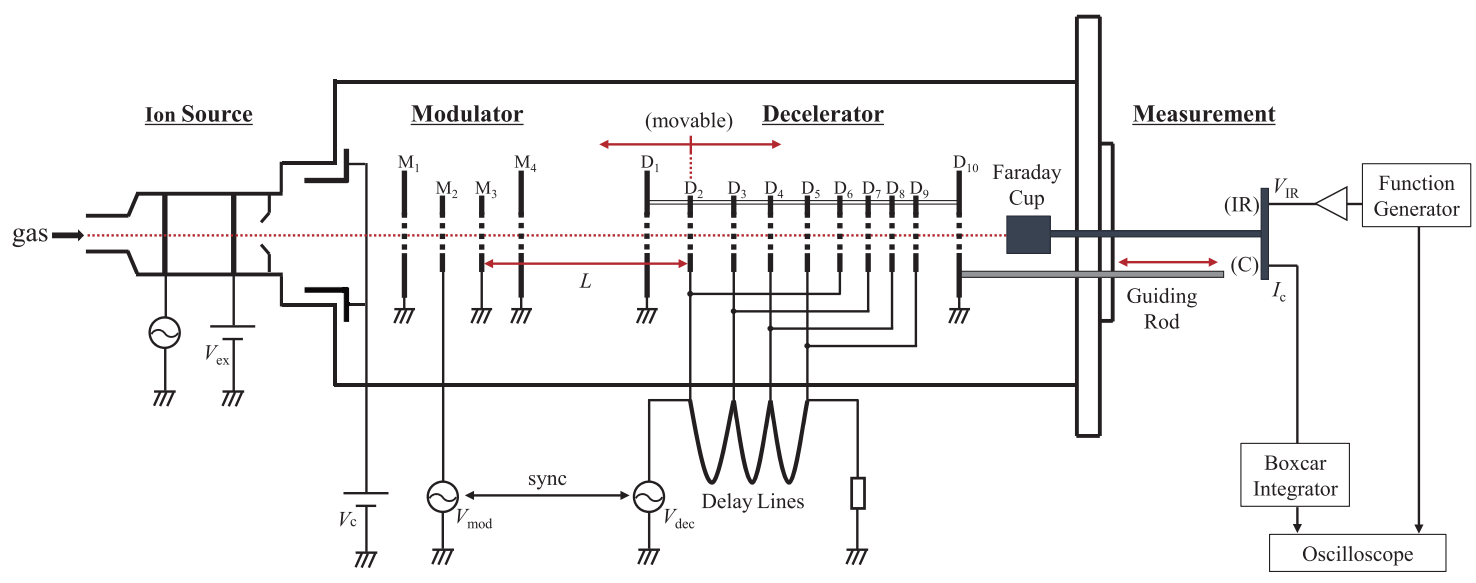

Fig. 1 Schematic view of the experimental device.

trode is set in the downstream of the extraction electrode to suppress diffusion of the extracted ion beam by an application of convergence voltage $\left(V_{\mathrm{c}}\right)$.

The modulator consists of four grid electrodes. The modulation voltage $\left(V_{\mathrm{mod}}\right)$ of $7 \mathrm{MHz}$ is applied to the electrode $\mathrm{M}_{2}$, and other electrodes $\left(\mathrm{M}_{1}, \mathrm{M}_{3}, \mathrm{M}_{4}\right)$ are grounded. In this condition, standing wave field (SWF) is created in the region between $M_{1}$ and $M_{2}$ and that between $M_{2}$ and $M_{3}$. The extracted helium ions are velocity modulated by these electric fields. The intervals between the electrodes are $14 \mathrm{~mm}$ which is equivalent to a quarter of the wavelength of the traveling wave for the beam extracted with $V_{\text {ex }}=3.2 \mathrm{kV}$.

The decelerator consists of ten grid electrodes. The first electrode of $D_{1}$ and the tenth electrode $D_{10}$ are grounded, and the deceleration voltage $\left(V_{\mathrm{dec}}\right)$ is applied to other electrodes $\left(\mathrm{D}_{2}-\mathrm{D}_{9}\right)$. In an actual TWDEC, $V_{\mathrm{dec}}$ is induced by incident bunched beam. In the simulation experiments, however, $V_{\mathrm{dec}}$ is applied externally because the beam is too weak to induce strong field enough to beam deceleration. This scheme is called active decelerator [3]. $V_{\text {dec }}$ is supplied by an RF source synchronized with $V_{\text {mod }}$ via coaxial delay lines which have terminals to connect the electrodes at every $\pi / 2$ phase positions. The electrode $\mathrm{D}_{i}$ and $\mathrm{D}_{i+4}(i=2,3,4,5)$ are connected, respectively, and voltages of the same amplitude are applied although they have some errors to the ideal values, and the details are presented in the previous paper [2]. The decelerator is designed based on the constant deceleration scheme, the details of which are also presented in Ref. 2. In this study, a new function is introduced that this set of decelerator electrodes is movable in the axial direction. We can change the distance between the modulator and the decelerator from the outside of the metal chamber by using a guiding rod. We define $L$ as a distance between $\mathrm{M}_{3}$ and $\mathrm{D}_{2}$.

In the measurement section, a Faraday cup is installed at the end of the device. It consists of an ion repeller grid (IR), a secondary electron repeller grid, and a collector plate $(\mathrm{C})$. The current of $\mathrm{C}\left(I_{\mathrm{C}}\right)$ is averaged by a boxcar integrator synchronized by plasma production RF pulses.
By sweeping the voltage of IR $\left(V_{\mathrm{IR}}\right), I_{\mathrm{C}}-V_{\mathrm{IR}}$ characteristic is obtained. An energy distribution $f(E)$ can be derived from the following equation:

$$
\left.f(E) \propto \frac{1}{\sqrt{V_{\mathrm{IR}}}} \frac{\mathrm{d} I_{\mathrm{C}}}{\mathrm{d} V_{\mathrm{IR}}}\right|_{V_{\mathrm{IR}}=\frac{E}{e}},
$$

where $E$ is ion energy and $e$ is unit charge.

The average energy of the ions can be obtained by

$$
\langle E\rangle=\frac{\int_{0}^{\infty} E f(E) \mathrm{d} E}{\int_{0}^{\infty} f(E) \mathrm{d} E} .
$$

Deceleration efficiency can be defined by

$$
\eta_{\mathrm{dec}}=\frac{\left\langle E_{0}\right\rangle-\langle E\rangle}{\left\langle E_{0}\right\rangle},
$$

where $\left\langle E_{0}\right\rangle$ means the average energy of incident beam.

We examined $\eta_{\text {dec }}$ for eight kinds of phase difference between $V_{\text {mod }}$ and $V_{\text {dec }}$ for one case, and the largest $\eta_{\text {dec }}$ was taken for evaluation.

\section{Results and Discussion 3.1 Experimental results}

The measurement conditions are $V_{\mathrm{ex}}=3.2 \mathrm{kV}, V_{\mathrm{c}}=$ $520 \mathrm{~V}, V_{\text {mod }}=200 \mathrm{~V}_{0 \mathrm{p}}\left(\mathrm{V}_{0 \mathrm{p}}\right.$ : zero-to-peak voltage $)$, and $V_{\mathrm{dec}}=150 \mathrm{~V}_{0 \mathrm{p}}$, where the value of the deceleration voltage amplitude is that on $\mathrm{D}_{5}$ and $\mathrm{D}_{9}$. We examined energy distribution by changing $L$. In the present experimental conditions, $L$ corresponding to the bunching length is about $24 \mathrm{~cm}$ which is confirmed by numerical calculation shown in Sec. 3.3.

Figure 2 shows examples of measured $f(E)$ by solid curves. Here, (a) and (b) are for $L=16 \mathrm{~cm}$ and $36 \mathrm{~cm}$, respectively. In the figure, $f(E)$ of the incident beam, that is, $V_{\text {mod }}=V_{\text {dec }}=0 \mathrm{~V}_{0 \mathrm{p}}$ is also indicated by dashed curves, showing that $\left\langle E_{0}\right\rangle$ is found around extracting energy of $e V_{\mathrm{ex}}=3.2 \mathrm{keV}$. Note that the ordinate value for the incident beam is scaled by $1 / 3$.

According to Fig. 2, there are both decelerated (lower than $3.2 \mathrm{keV}$ ) and accelerated (higher than $3.2 \mathrm{keV}$ ) ions 


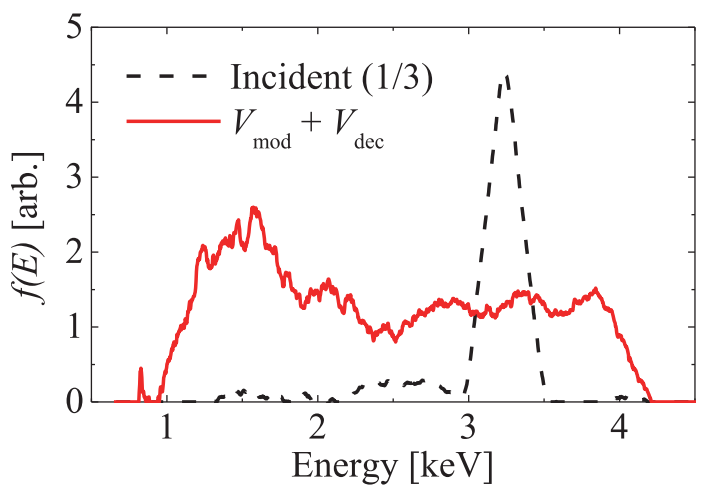

(a) $L=16 \mathrm{~cm}$

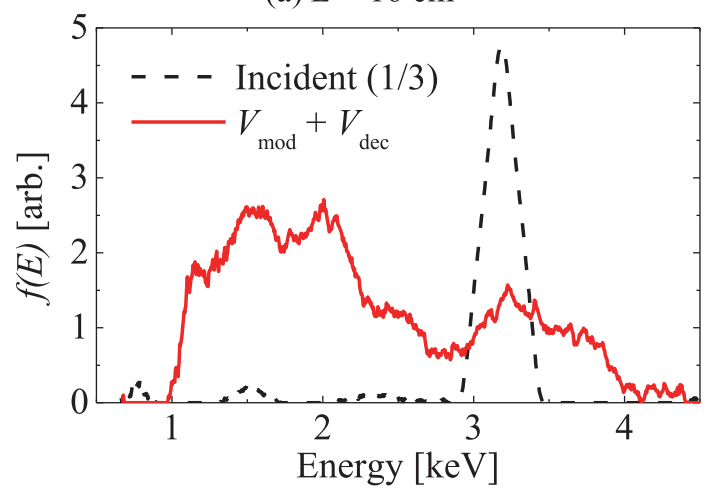

(b) $L=36 \mathrm{~cm}$

Fig. 2 Measured energy distribution functions.

when $V_{\text {mod }}$ and $V_{\text {dec }}$ are applied. The decelerated ions are more than accelerated ions, thus the average energy decreases due to operation of TWDEC. Comparing (a) and (b) in Fig. 2, $f(E \sim 2 \mathrm{keV})$ for (b) is larger than that for (a), and $f(E \sim 4 \mathrm{keV})$ for (b) is smaller than that for (a). This means that deceleration effect varies according to the axial position of the decelerator and that for $L=36 \mathrm{~cm}$ is superior to that for $L=16 \mathrm{~cm}$.

\subsection{Results of numerical calculation}

We also examined deceleration effects by using numerical calculations which was one-dimensional particle orbit calculation. In the calculation model, particles are affected only by electric field due to electrode voltages, and space charge effect is not taken into account. The details are explained in Ref. 4. The conditions of $V_{\mathrm{ex}}, V_{\mathrm{mod}}$, and $V_{\text {dec }}$ are the same as those in the simulation experiment.

Figure 3 shows calculated energy distribution functions for the cases of (a) $L=16 \mathrm{~cm}$ and (b) $L=36 \mathrm{~cm}$. Comparing (a) and (b) in Fig. 3, the energy distribution in $E<2.5 \mathrm{keV}$ for (b) is more than that for (a), and vice-versa in $3.5 \mathrm{keV}<E<4 \mathrm{keV}$. These results are well corresponding to the experimental results in Fig. 2 while ions around $3.2 \mathrm{keV}$ are quite few in Fig. 3. This difference around extracting energy comes from the phenomenon that the insufficient modulation results in the remained component of the incident beam in the experiment, and it was observed in the previous experiments [2]. According to the previ-

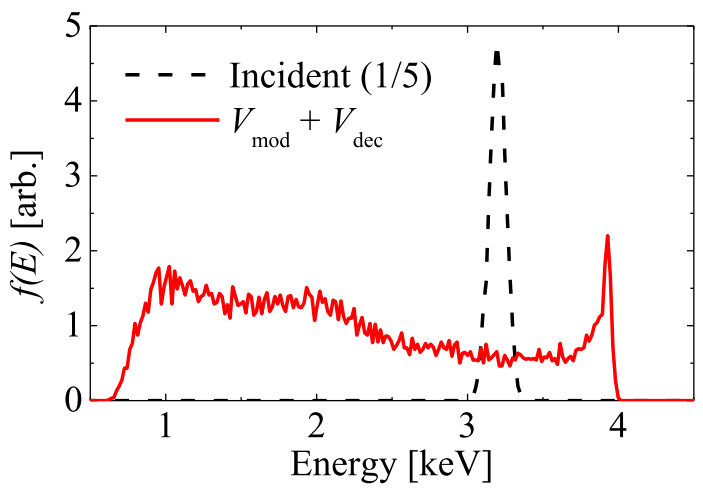

(a) $L=16 \mathrm{~cm}$

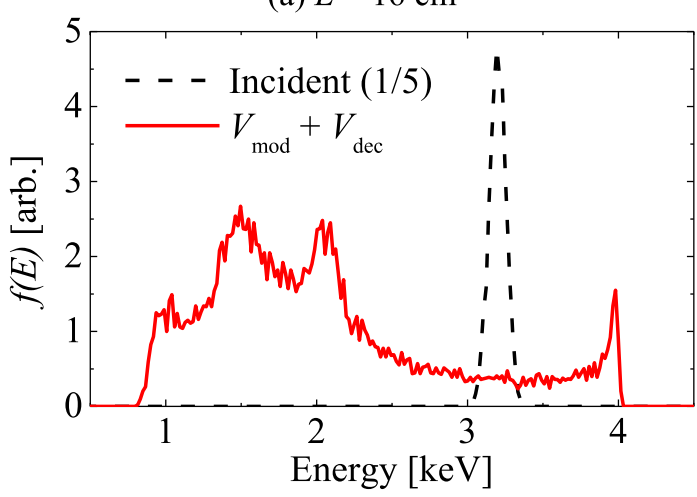

(b) $L=36 \mathrm{~cm}$

Fig. 3 Calculated energy distribution functions.

ous research [5], this phenomenon depends on time and insufficient modulation reduces with the lapse of $V_{\text {mod }}$ application time. In the present experiment, we extended the pulse width for $V_{\text {mod }}$, so the remained component was significantly reduced.

\subsection{Evaluation by deceleration efficiency and discussion}

Deceleration efficiency was evaluated according to Eq. (3). Figure 4 shows deceleration efficiency versus $L$ for experimental result. The values are averaged ones for five times measurement and the error bars represent the standard deviation. The maximum $\eta_{\mathrm{dec}}$ of $26.4 \%$ is obtained at $L=32 \mathrm{~cm}$, which is much higher than about $24 \%$ around bunching length.

We also examined by numerical calculation. In Fig. 5, deceleration efficiency and axial ion distributions as functions of $L$ are shown, where $L$ means both distance between the modulator and the decelerator, and axial position from the modulator electrode $\mathrm{M}_{3}$. As for the axial ion distributions, those just by modulation field application for eight different RF phases are shown, and they mean snap shots with an interval of $1 / 8$ of RF period. The highest ion density is obtained for $\pi / 2$ phasing at $L=23.8 \mathrm{~cm}$. As the calculation employs randomness in the energy distribution of incident ions, the bunching position has ambiguity and is estimated to be $23.8-24.5 \mathrm{~cm}$. As for deceleration efficiency, $\eta_{\text {dec }}$ was calculated by using Eq. (3) where numer- 


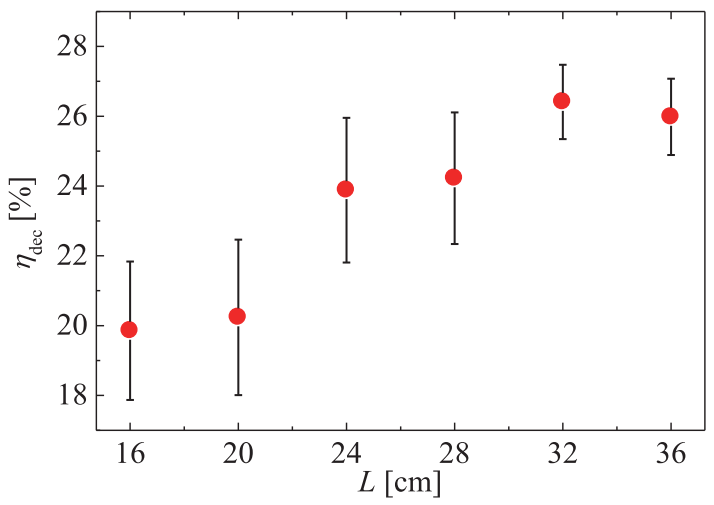

Fig. 4 Deceleration efficiency versus distance between the modulator and the decelerator for experiment.

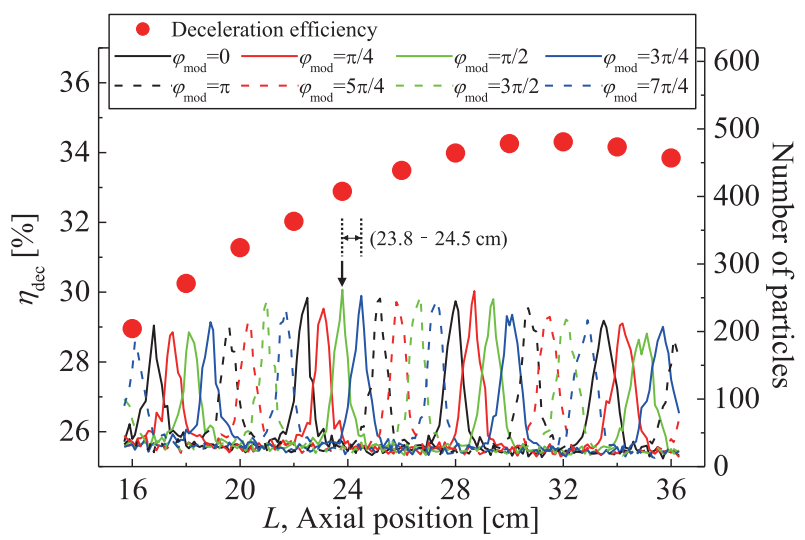

Fig. 5 Deceleration efficiency and ion distribution as functions of $L$ for numerical calculation.

ically calculated average energy of ions is used. The tendency of variation is quite similar to that for experiment in Fig. 4. The maximum value is at $L=32 \mathrm{~cm}$ which is the same as that for the experiment. However, $\eta_{\text {dec }}$ is higher than that for the experiment by approximately $8 \%$. This is due to above-mentioned remained component of incident beam observed in the experiment. These results show that the bunching position is not necessarily the optimum one for efficiency and the optimum $L$ is $32 \mathrm{~cm}$ in the condition of $V_{\text {mod }}=200 \mathrm{~V}_{0 \mathrm{p}}$ and $V_{\mathrm{dec}}=150 \mathrm{~V}_{0 \mathrm{p}}$.

The optimum positions for other conditions were also examined by numerical calculation. The results for the similar structure as the experiment are summarized in Fig. 6. The bunching length is determined by $V_{\text {mod }}$ (abscissa) and its ambiguity is expressed by an error bar. The position of the decelerator giving the maximum efficiency depends on $V_{\text {mod }}$ and $V_{\mathrm{dec}}$, and it is different from the bunching position for both cases of $V_{\mathrm{dec}}=150 \mathrm{~V}_{0 \mathrm{p}}$ and $230 \mathrm{~V}_{0 \mathrm{p}}$. Thus, the bunching position is not necessarily the optimum one for efficiency. This is completely different from conventional expectation.

This may be because that the decelerator is designed based on the constant deceleration scheme, which can de-

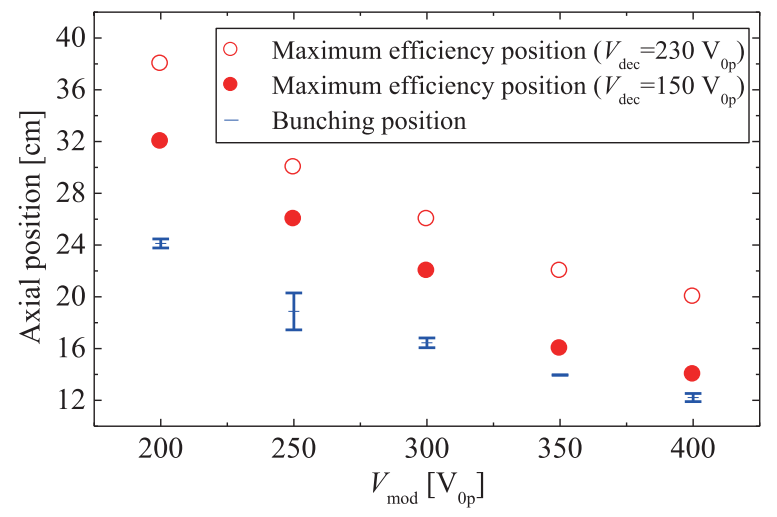

Fig. 6 Bunching and maximum efficiency positions for some conditions of $V_{\text {mod }}$ and $V_{\mathrm{dec}}$.

celerate ions in a trapping region of the phase space [6]. According to Fig. 5, dully bunched peaks are in the downstream. Ions in those peaks can be decelerated if the trapping region is wide. Total number of those ions is more than those in the peak at the bunching position. Thus, the maximum efficiency position of the decelerator may be far from the bunching position. The quantitative analysis should be a future work because it is difficult to obtain final energy of ions as the bouncing motion is interrupted at the end of the decelerator with a short length.

\section{Conclusion}

In this study, we investigated the dependence of deceleration efficiency on the axial position of the decelerator by using simulation experiments and numerical calculations. In the experiment, the maximum deceleration efficiency is obtained at a different position from the bunching position. This is confirmed by numerical calculation including different working parameters cases. Thus, the bunching position is not necessarily the optimum one for efficiency and this is different from conventional expectation. The detailed analysis on the constant deceleration scheme is necessary in future.

\section{Acknowledgment}

The authors acknowledge valuable discussions with Drs. J. Miyazawa and T. Goto. This work was supported in part by the bilateral coordinated research between Plasma Research Center, Univ. Tsukuba, National Institute for Fusion Science, and Kobe Univ. (NIFS13KUGM082).

[1] H. Momota, LA-11808-C, Los Alamos Natl. Lab. 8 (1990).

[2] Y. Togo et al., Plasma Fusion Res. 10, 3405013 (2015).

[3] H. Takeno et al., Fusion Eng. Des. 83, 1696 (2008).

[4] H. Takeno et al., Jpn. J. Appl. Phys. 39, 5287 (2000).

[5] H. Takeno et al., The 10th Joint Conference on Fusion Energy, 20-130 (2014) (in Japanese).

[6] H. Katayama and K. Sato, J. Plasma Fusion Res. 77, 698 (2001) (in Japanese). 